\title{
Wet wipes and disposable surgical masks are becoming new sources of fiber microplastic pollution during global COVID-19
}

\author{
Tong $\mathrm{Hu}^{1,2} \cdot$ Maocai Shen ${ }^{1,2} \cdot$ Wangwang Tang ${ }^{1,2}(\mathbb{D}$
}

Received: 22 June 2021 / Accepted: 3 November 2021 / Published online: 10 November 2021

○ The Author(s), under exclusive licence to Springer-Verlag GmbH Germany, part of Springer Nature 2021

\begin{abstract}
The demand of wet wipes and masks has been rising worldwide since the outbreak of global COVID-19; however, with more reports about improper handling of wipes and masks, their potential threats to the environment are gradually emerging. Wipes and masks are made of a large number of plastic fibers, which are easily broken and fragmented into microplastic fibers under the influence of environmental factors. Weathered wipes or masks can release billions of microplastic fibers, which is a great challenge to the local ecological security. Wipes and masks as new microplastic pollution sources and their potential role in the ecosystem have not been fully recognized and considered. Microplastic fiber pollution is a huge environmental issue, and how to prevent a large number of discarded wipes and masks from entering the environment and how to deal with them are an important issue for all countries and regions in the world. In the post era of global COVID-19, disposable wipes and masks, as new sources of environmental microplastic fiber pollution, should be given concern. It is urgent to recognize this potential environmental threat and prevent it from becoming the next microplastic problem.
\end{abstract}

\section{Keywords}

Microplastics, Wet wipes, Disposable surgical masks, COVID-19, Waste management

\section{Concerns of global fiber microplastic pollution}

As an emerging environmental pollutant, microplastics have been attracting the public attention (Thompson et al. 2004; Shen et al. 2020). With the rise of the "plastic" era, a large of number of microplastics and even nanoplastics enter the environment, which makes microplastics continue to accumulate in the environment and cause potential harms to the ecosystem (Bouwmeester et al. 2015; Browne et al. 2011).

Responsible Editor: Philippe Garrigues

Wangwang Tang

wtang@hnu.edu.cn

1 College of Environmental Science and Engineering, Hunan University, Changsha 410082, China

2 Key Laboratory of Environmental Biology and Pollution Control (Hunan University), Ministry of Education, Changsha 410082, China
At the same time, microplastics can change the environmental behavior of pollutants by adsorbing a large amount of heavy metals (Hodson et al. 2017; Koelmans et al. 2016; Shen et al. 2021a), persistent organic pollutants (Koelmans et al. 2013; Velzeboer et al. 2014) and microorganisms (Kirstein et al. 2016; Shen et al. 2019a) in the environment, further affecting environment health. In 2015, the problem of microplastic has been listed as the second largest scientific problem in the field of environmental and ecological sciences (Horton et al. 2017).

Fiber microplastics have been proved to be the main microplastic pollutants in water environment in a lot of previous studies, and the main components of microfibers are polyamide (PA), polyethylene terephthalate (PET), polyethylene (PE) and polypropylene (PP), respectively (Briain et al. 2020; Lares et al. 2018). In addition to the weathering and degradation of fishing line and nets discarded in the environment, these microfibers come from the shedding of fine fibers in the washing process of synthetic fiber fabrics. At present, the global emission of microplastic pollution is about 0.8-2.5 million tons per year, of which the shedding microfibers from textiles account for about $34.8 \%$ ( 0.52 million tons) (Xu et al. 2020). It is estimated that about $7 \times 10^{5}$ microfibers will be released from each $6 \mathrm{~kg}$ synthetic fabric washed by a household washing machine (Napper and Thompson 2016). Due to the influence of season, detergent, 
water quality, clothing freshness and other factors on the release of fiber microplastics during laundry (De Falco et al. 2018), it is difficult to control and estimate the fiber microplastics. Fiber microplastics are released in the whole life of cycle of textile production and the whole process of textile use and final disposal (Murphy et al. 2016). Additionally, cigarette butts, made of cellulose acetate plastic and usually composed of 15,000 strands of fibers, are also a potential source of fiber microplastics in the environment (Novotny et al. 2009). At present, global tobacco consumption has reached about 6 trillion cigarettes per year, and it is estimated that three-fourths of cigarette butts have not been properly handled (Belzagui et al., 2021). Accordingly, about $8.45 \times 10^{5}$ tons (5.6 trillions) of cigarette butts are discarded in the world every year (Parker and Rayburn 2017). Therefore, these potential sources of fiber microplastics in the environment must be vigilant and controlled.

Unfortunately, a new source of fiber microplastics in the environment is coming quietly. Since the outbreak and spread of COVID-19, the consumption of protective products such as disposable wet wipes and disposable surgical masks has increased sharply. It is estimated that there are about 129 billion masks used every month in the world during COVID-19, most of which are disposable masks made using microplastic fibers (Prata et al. 2020). Disposable surgical masks are made of PP fibers (Aragaw 2020), which is a kind of microplastic commonly found in the environment. The public does not know that disposable wet wipes are mostly made of plastic and cannot be degraded in the environment. These wipes are thrown away in the environment after use by the way of discarding the facial tissue. Once in the environment, these discarded masks and wipes will be vulnerable to radiation and heat, and the fiber microplastics will be released from the matrix. Fiber microplastics are more likely to break in the environment, thereby resulting in the release of a large amount of secondary microplastics, which further aggravates the pollution of microplastics. If not properly collected and managed, wipes and masks can be transported from land to freshwater and marine environments via surface runoff, wind and human activities (Han and He 2021; Saliu et al. 2021). Like other plastic fragments and microplastics, wipes and masks may accumulate and release harmful chemical substances, such as bisphenol A, dye and heavy metals (Sullivan et al. 2021). A new and bigger problem is that wipes and masks are made from plastic fibers. When decomposed in the natural environment, wipes and masks can release more microplastics (Wang et al. 2021), which are easier and faster than plastic bags and boxes (Ma et al. 2021; Shen et al. 2021b). Furthermore, the new generation of nano masks uses nano-sized plastic fibers and adds new sources of nanoplastic fiber pollution, which may worsen the effect (Xu and Ren 2021). Indeed, wet wipes would only cause microfiber pollution to a low extent, but a large number of discarded wet wipes into the environment is also a problem that cannot be ignored during COVID-19. Meanwhile, limited information on the degradation of wipes and masks in the environment can be obtained. With more and more reports about improper handling of masks and wipes, it is urgent to recognize this potential environmental threat and prevent it from becoming the next microplastic problem. The aim of this paper is to evaluate the potential sources of discarded wipes and masks as environmental fiber microplastics and the potential ecological effects of the released microplastic fibers on the ecosystem.

\section{Are wet wipes and disposable surgical masks a new problem to the environment?}

The white pollution caused by plastics has become a "stubborn disease" of the environment, and the harm is still expanding. In recent years, especially since COVID-19 outbreaks, the consumption of disposable wet wipes and disposable surgical masks has dramatically increased (World Health Organization 2020). The main components of personal protective products on the market, such as wipes and masks, are mostly nonwoven fabrics, which are based on plastic fiber (Briain et al., 2020). Due to the fact that the ingredients of wipes and most masks are only marked as "nonwoven" in the packaging, the public is not aware of the ingredients of wipes, masks and other protective articles on the market. Notably, new application of nonwoven fabrics may bring new challenges. Recently, the release mechanisms and influencing factors of microplastics from disposable medical masks have been thoroughly discussed (Sullivan et al. 2021; Wu et al. 2021). However, limited information can be available on the release of microplastic fibers from wet wipes. The fibers in wipes are not arranged closely and are easy to be separated from the matrix under the action of external force. First of all, the wipes seem to be an improvement on the old toilet paper, but they will bring a lot of problems to the sewer system. The public is less aware that toilet paper is made of wood pulp mixed with other materials and is made extremely thin. The purpose of this is to make it rot when it meets water and some of it even dissolves in water, so as not to block the downpipe. After that, the domestic sewage is transported to the sewage treatment plant, where the sewage will reach the discharge standard through a series of treatment. However, wet wipes are different, and they are made of nonwovens, fabrics, dust-free paper or other raw materials as carriers, purified water as production water and appropriate amount of preservatives and other auxiliary materials. Therefore, they are not easy to rot in water and are not easy to be degraded by microorganisms. In real life, people do not realize that wet tissue should not be washed down into the toilet, and it is easy to throw it directly into the sewer 
like toilet paper. But in fact, just a small amount of wipes can clog drains and sewers. Once these wipes are mixed with fat or oil, the subsequent blockage in the sewer becomes large, which narrows the pipe and gradually blocks it (Briain et al. 2020). Even though it is flushable, it still can cause serious environmental pollution. The research clearly showed that these nonwovens are not easy to decompose with the disturbance of water body and are not suitable for flushing toilets (Khan et al. 2019). Wipes can be broken because of mechanical mixing defects, residual stress and absolute stress in the wastewater treatment plant (Enfrin et al. 2020). Wet wipes and other macro debris can cause operational problems in the sewer system by forming long fibers. These structures block the normal sewage channels and have adverse influences on the microbial community and biological processes in the wastewater treatment systems (Briain et al. 2020). The most worrying thing is that the public do not pay as much attention to the current wipes' pollution as they do to the "white pollution". The treatment of this pollution level is no less than the high pollution of plastic products, just like the treatment of degradable paper towel, randomly discarded, randomly landfilled and randomly disposed.

Additionally, the outbreak of COVID-19 renders the recovery of disposable plastic products, disposable surgical masks, in the world. Evidence has shown that a large number of disposable surgical masks are randomly discarded in the city lanes, blocking the sewage pipes, thus aggravating the current environmental challenges (Aragaw 2020). These masks inside the waste surface are transported to the water body through surface runoff and wind (Fadare and Okoffo 2020). As a result of the emergence of COVID-19, the public use mask extensively, which accelerates the environment disorder in the land and water environment, and the global pandemic has not reduced the challenge of microplastic pollution in the environment. Fig. 1 showed the discarded disposable wet wipes and surgical masks in the wild during COVID-19. Disposable surgical masks, as a new source of fiber microplastics, first enter the environment, such as
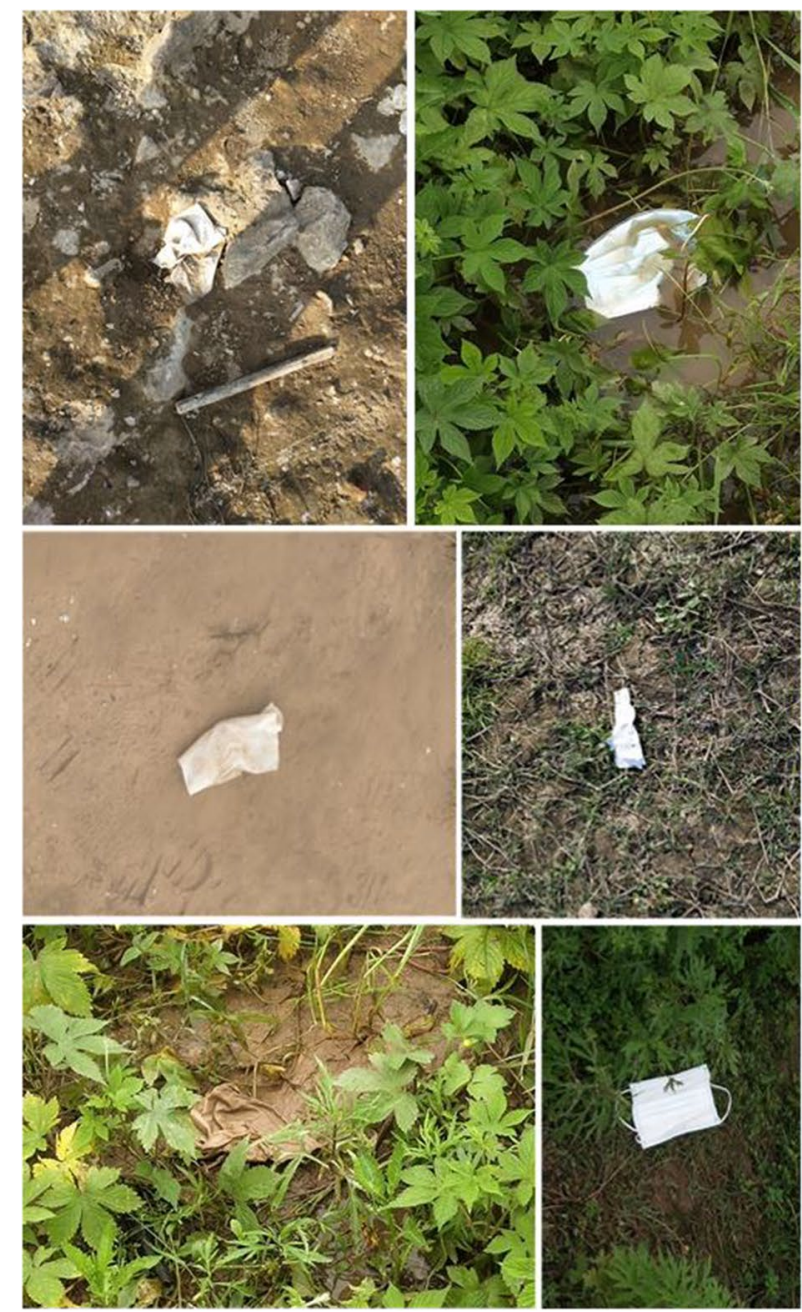
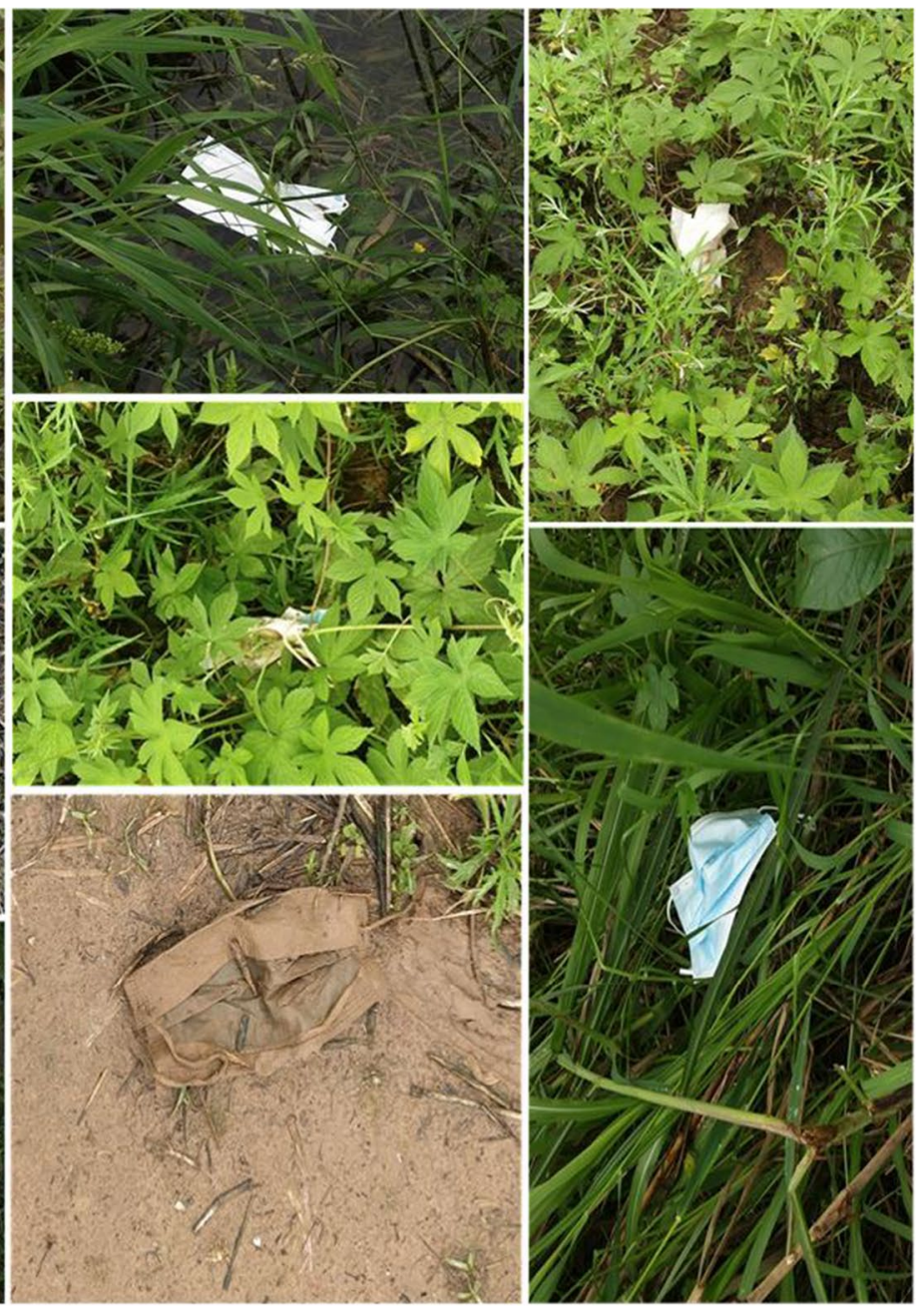

Fig. 1 Discarded disposable wet wipes and surgical masks found in the wild during COVID-19 
landfill of garbage in public places, and then go into freshwater and oceans. Evidence demonstrated that these masks can be degraded into microplastic particles under various environmental conditions (Schmidt et al. 2018). In addition to the effect of masks as a new source of fiber microplastic pollution, masks can also be used as medium for the outbreak of COVID-19 (Shruti et al., 2020). Due to the nature of plastic, it is easy for microorganisms to settle and reproduce on the surface, which can spread or attack along the food chain (Shen et al. 2019b). Compared with the natural microbial communities in the surrounding aquatic environment, there may be a significant difference in microbial components (Yang et al. 2020), which will affect the microbial habits and environmental processes in aquatic ecosystems. The outbreak of COVID-19 has led to a sharp increase in the abundance of masks and wipes in the global environment, which has questioned the impact of the ecological functions on the whole ecosystem and animal health.

Moreover, these discarded wipes and masks in the environment will become fiber microplastics over time. Fig. 2 illustrated the fates and behaviors of discarded wipes and masks in the environment. Microplastics are often swallowed by aquatic organisms, including fish (Tien et al. 2020) and seafood (Sun et al. 2018; Sussarellu et al. 2016). Marine organisms often starve to death after eating plastic debris such as wipes or discarded plastic bags (Hu et al. 2019). It has been reported that in 2020, about 52 billion masks have been produced worldwide, of which 1.56 billion entered the oceans through various pathways. Each mask is about $3-4 \mathrm{~g}$ in weight, and after conversion, there are about 4680-6240 tons of discarded masks in the ocean. A research conducted by Everaert et al. (2018) proposed that the safe concentration of microplastics in the marine environment can be as high as 6.55 items $\mathrm{L}^{-1}$. However, the discarded wipes and masks may cause a sharp rise in the abundance of microplastics in the local environment, because a completely weathered wipe or mask may release billions of microplastic fiber to the environment. Many of the above facts have proved that the problem of wipes and masks may be more significant than ever. Do we really want to exchange our health and environmental pollution for this temporary convenience? The answer is NO! The most urgent thing is to raise the public vigilance and let them know that discarded personal protective equipment should be handled correctly. Under the guidance of government departments, people gradually realize the correct treatment, and this silent "new plastic pollution" can be quietly annihilated in daily life.

\section{What are the effects of wet wipes and disposable surgical masks on humans?}

Discarded wipes and masks have respiratory secretions that contain many bacteria, even harmful pathogens. Once in the environment, the rapid spread of wipes and masks may cause the spread of harmful pathogens in large areas (Shen et al. 2019c). In addition, chemical problems of additive leaching, like bisphenol A, in daily plastic products have been proved (Huang et al. 2012). However, it is more challenging to understand the threat of microplastics and nanoplastics released by wipes and masks to human beings and take appropriate actions against this

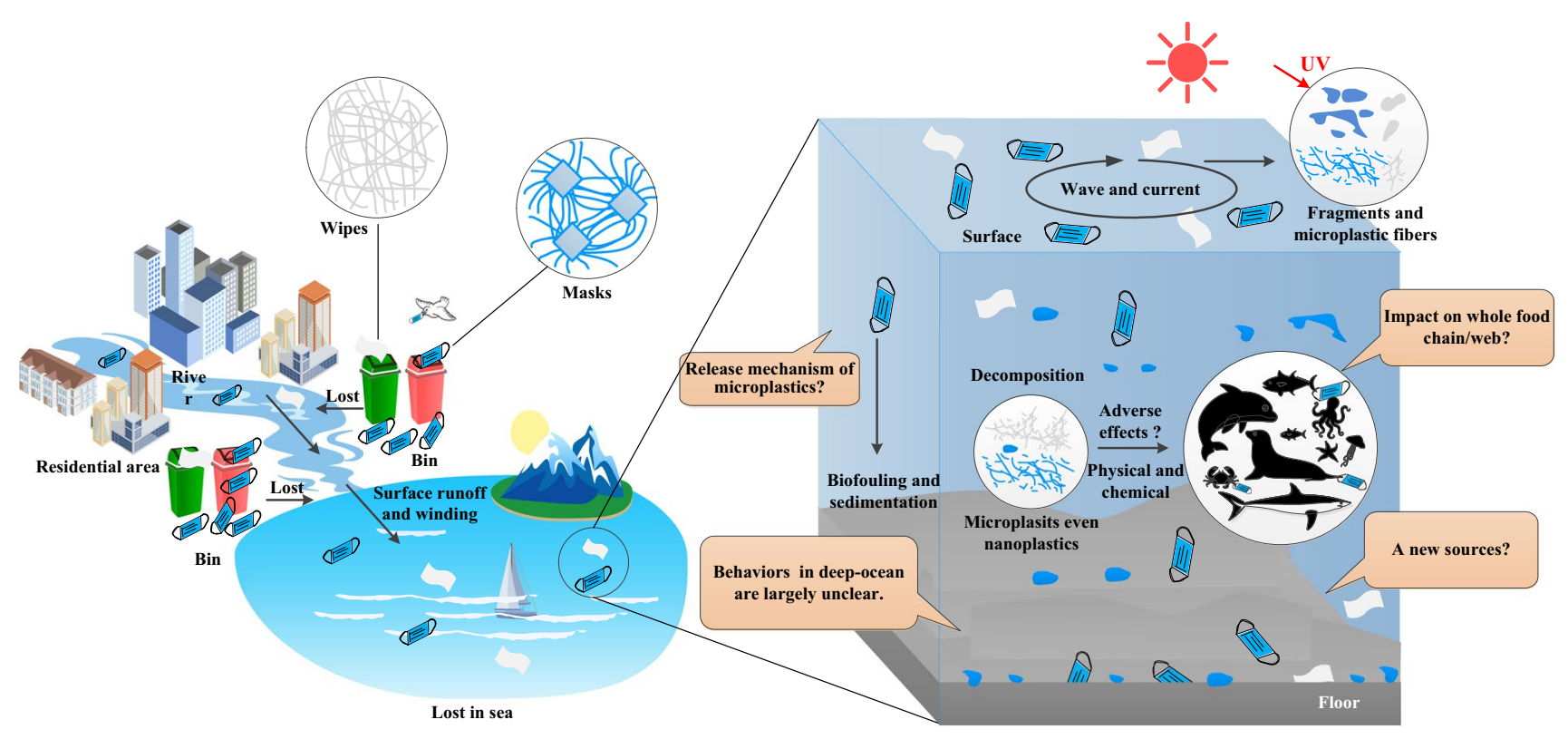

Fig. 2 Fates and behaviors of discarded wipes and masks in the environment 
knowledge. Although a large amount of wipes and masks enter the environment, so far, the research on their natural degradation in the environment and the release mechanisms of microplastic and nanoplastic fibers is very limited. Moreover, there is a lack of comparative study on the relative harm of microplastics and non-plastic particles to human body. In all the inhaled and ingested, it is possible that the nanoplastics with an average size less than 1 $\mu \mathrm{m}$ can pass through the epithelial lining of the lung and gastrointestinal tract (Shen et al. 2019d; Wright and Kelly 2017). Ingestion of microplastics poses a further threat to human health; however, it has not been quantified. The presence of microplastics in human feces has been confirmed (Schwabl et al. 2019), and a research showed that up to 52,000 microplastics can be ingested by US citizens every year (Cox et al. 2019). A recent study carried by Ragusa et al. (2021) reported that microplastic particles were found in all placental portions, indicating that microplastics have potential threat to the fetal health.

At present, trace of microplastics has been found in a variety of human foods, including seafood ( $\mathrm{Li}$ et al. 2016; Vandermeersch et al. 2015), sea salt (Iniguez et al., 2017; Yang et al. 2015), drinking water (Koelmans et al., 2019; Mintenig et al. 2019; Oßmann et al. 2018; Shen et al. 2021c), beer (Gerd and Elisabeth, 2014), sugar and honey (Gerd and Elisabeth 2013, 2014). Evidence showed that damage was caused by oxidative stress in cerebral and epithelial human cell lines as observed in vitro research using polyethylene and polystyrene microplastics (Waring et al. 2018). But further damage would not occur because the particle sizes of microplastics limit their transfer in tissues. Paracellular uptake also may occur across the intestinal epithelium cells. Human colonic mucosal tissue model showed lower membrane transport efficiency $(<0.1 \%)$ for microplastics with $3 \mu \mathrm{m}$ (Yoo et al. 2011). However, for patients with intestinal diseases, the transport efficiency of microplastics would significantly increase due to changes in tissue permeability caused by inflammatory infection. In addition, evidence showed that nanoplastics can be able to penetrate cell membranes and placenta, inducing inflammation, oxidative stress and cell damage (Vethaak and Leslie 2016). Up to now, available data on microplastics contained in seafood and other food resources are not sufficient enough to evaluate the potential risks of microplastics for human health along the food chain (Yoo et al. 2011). Microplastics are difficult to enter the deep tissues and be quickly cleared by spleen (Bouwmeester et al. 2015). With the global influx of discarded wipes and masks, the content of microplastics and nanoplastics in environmental media may increase significantly. It is also urgent to develop a method for accurately evaluating the number of microplastic particles in food and the number of particles transferred along the food chain.

\section{Are fibrous microplastics caused by wipes and masks an issue relative to other types of microplastics?}

As we all know, microplastic fiber is an important part of microplastic pollution in the environment. However, evidence has demonstrated that the actual pollution of fiber microplastics to water environment is suspected to be much higher than that of other microplastic particles (Woods et al. 2018; Zhang et al. 2020). Although microplastic fibers are dominant in water environment, little attention has been paid to them so far. The global outbreak of COVID-19 has pushed the potential environmental sources of microplastic fibers, wipes and masks to the public attention. Wipes and masks are made of plastic fibers, which is different from other plastic products such as plastic bags and bottles. Plastic fibers are more likely to be broken and disintegrated under the influence of weathering factors such as heat and radiation in the environment, resulting in the release of great microplastic fibers (Xu and Ren 2021). A fully weathered mask can release more than several billions of microplastic fibers to the environment. Therefore, the degradation process of wipes and masks in the environment, the contribution to the environmental microplastic pollution and the release mechanism of microplastic fibers need to be explored timely. The outstanding nature of microplastic fibers and its high abundance in some areas make it necessary to consider its toxicity in the risk assessment of microplastic pollution. As a matter of fact, the absorption of microplastics depends on the concentration, bioavailability and degradation state of the fiber in the environment. Recently, Rebelein et al. (2021) systemically reviewed the toxic effects of underestimated environmental risk on organisms of microplastic fibers, and the authors suggested that microplastic fiber should be included in prospective monitoring studies to discuss appropriate methods and recommend exposure studies and risk assessment for these underrated contaminants.

Additionally, a large number of wipes and masks debris will be sent to the wastewater treatment plant through the sewage. The used flushable wipes will greatly increase the concentration of plastic fibers in sewage (Briain et al. 2020). Because of the high aspect ratio of fiber microplastics, it is easy to knot in sewage to block the sewage pipe. Compared with granular and ball microplastics, fiber microplastics have low size and other shape characteristics, which makes them more prone to the fragmentation and causes serious potential ecological hazards. Although the existing research on the removal of non-fiber microplastics has a certain reference value, the difference of interface interaction caused by different shapes may lead 
to the decrease of removal efficiency when applied to fiber microplastics. Consequently, the "cunning" fiber microplastics need to be regarded as a kind of independent microplastic pollutants. It is necessary to further improve its monitoring mechanisms, toxicological mechanism, interface mechanisms, removal mechanism and so on to supplement the existing gap in this field, especially after a large number of wipes and masks have entered the environment.

Garbage is a resource in the wrong place, which is also applicable to fiber microplastics and discarded wipes and masks. The global COVID-19 and environmental mask and wipe pollution provide a new opportunity to change social behavior and to take advantage of the current unprecedented participation in environmental issues and concerns in order to reduce the "discard" culture and rectify poor waste management, as well as to raise awareness of other potentially greater environmental issues.

\section{How and what can we do?}

At present, the epidemic is not over, and the use of masks and wipes will become the norm for a long time to come, and it will be very challenging and irresponsible to reduce the use of them indiscriminately. Methods and technologies for future research, control and removal of emerging new sources of microplastic contamination was proposed at Fig. 3. The rapid increase in the consumption and treatment of these disposable plastic products poses a potential threat to the environment; however, the impact on global plastic and microplastic pollution is basically unknown. Therefore, it is imperative to understand and mitigate these risks quickly. To obtain more information on the consequence of these disposable wipes and masks, environmental study and policy should be: a) To conduct interdisciplinary research on the environmental behavior and fate of disposable wipes and masks.

It is urgent to study the natural degradation and decomposition of disposable wipes and masks in the environment and the release of microplastics and even nanoplastics. At present, most of the reports about the environmental pollution caused by masks and wipes only stay at the macro level, that is, the impact on the beauty of the environment, but it did not go deep into the more serious fiber microplastic pollution. In addition, wipes and masks as an important carrier of environmental harmful chemicals and pathogens are also worthy of investigation.

b) To study composite pollution mechanism and influencing factors of fiber microplastics and pollutants.

At present, there are many reports about the physical damage of discarded masks and wipes to marine organisms, such as entanglement and blockage of esophagus. Perfluorinated compounds, polycyclic aromatic hydrocarbons, pesticides, heavy metals and other pollutants are easy to accumulate on the surface of microplastics. However, there is still a lack of systematic understanding of the transport, distribution and complex toxicological mechanism of fiber microplastics and their attached pollutants in marine organisms. There are still many questions about the joint toxicity of fiber microplastics and toxic pollutants to organisms. More attention should be paid to the transfer and enrichment of fiber microplastics in the food web brought by wipes and masks. It is imperative to understand the accumulation of pollutants loaded on the surface of microplastics and the risk to human health. Moreover, the research on microplastic removal mechanisms can enable us to optimize the traditional process (e.g., coagulation, flocculation and membrane separation) or develop new technologies to achieve efficient removal of microplastics.

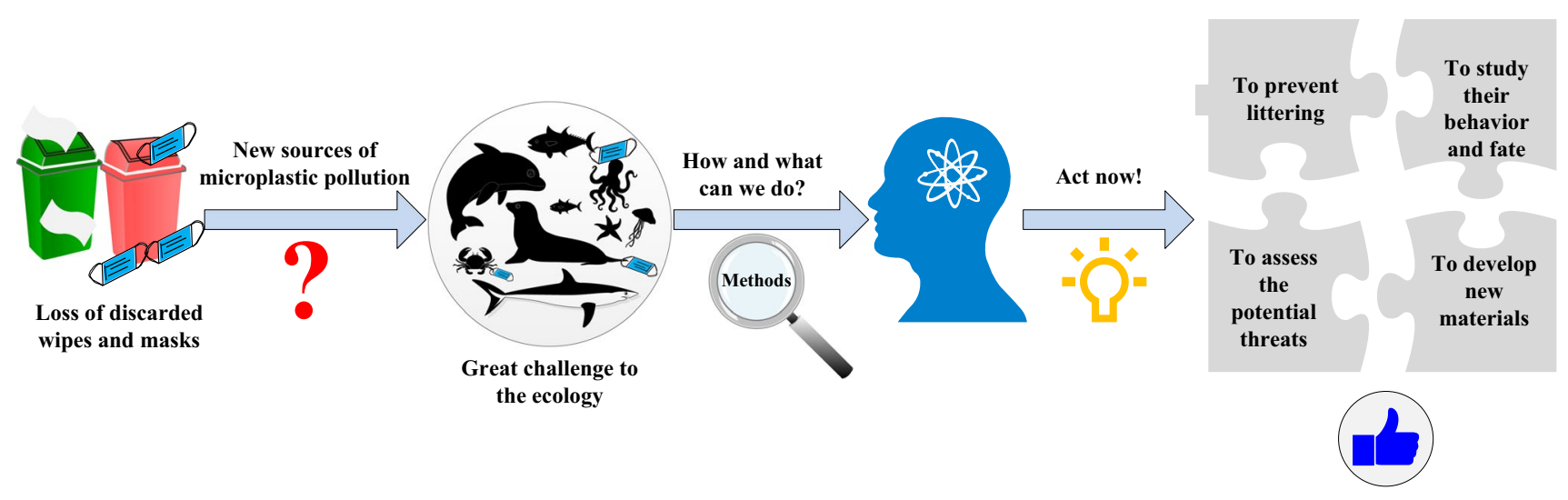

Fig. 3 Proposed methods for future research, control and removal of emerging new sources of microplastic contamination 
c) To reduce the "discard" culture and rectify poor waste management.

The root of plastic pollution is not the plastic itself, but the relationship between plastics and humans. At the level of government decision-making, it is necessary to establish the management, policies and regulations of disposable wipes and masks, promote local governments to publicize the environmental hazards of wipes and masks and microplastics and cooperate with the media to strengthen the public awareness of microplastics. At the local level, efforts are required to strictly supervise the collection and treatment of wipes and masks in each community, upgrade the removal equipment of microplastics in sewage treatment plant, train relevant technical personnel of mask and microplastic pollution management and strengthen the ecological restoration measures of microplastic pollution in typical areas. At the level of mass participation, we should improve the awareness of and actively publicize the pollution of wipes, masks and microplastics, and actively obey the relevant policies of local governments to reduce the discarding of wipes and masks.

d) To develop degradable wipes and masks to replace existing ones.

The government should make greater efforts to encourage enterprises to use degradable materials to replace the existing non-degradable plastics. Because of its special process, the toughness of the wipes is enhanced, so as to avoid adding plastics to the wipes and reduce the pollution caused by microplastics. Degradable natural materials also let us no longer worry about the non-degradable white pollution. The manufacture of biodegradable treatment masks and wipes can also be developed, but the higher cost and unknown safety of new materials need to be considered, which requires the government to give certain preferential policies and economic subsidies.

Nowadays, the research on the existence of plastics and microplastics in the environment, their impact on the whole ecosystem is extensive, but only through scientific practice cannot understand and change these big problems. To solve the problem of plastic and microplastic pollution needs large-scale political and economic changes, but this change must be based on sound objective science and social science. At present, there is a gap between scientific research and the complementary research needed to understand the social dimension of plastic pollution. Recognizing the importance of this knowledge gap, and narrowing it, is critical for us to reduce the amount of man-made wipes, masks and other plastic products and microplastics that persist in the environment.

\section{Conclusions}

The sudden outbreak of global COVID-19 has caused the disposable wet wipes and masks to enter the environment in large quantities, which masks the situation of plastic and microplastic pollution worse. Wipes and masks are made of plastic fibers, which is easier to be weathered in the environment than other plastic products such as plastic bags and bottles. Wipes and masks will gradually break down into pieces in the environment and eventually become microplastic fibers even nanoplastics. Weathered wipes or masks can release billions of microplastic fibers, which is a great challenge to the local ecological security. As users of wipes and masks and producers of contamination, each of us should be responsible for this. The global COVID-19 is not over yet, and it should not be to bring irreparable harm to the earth and lead to the next bitter fruit! However, it is still a long way to go in dealing with the pollution of wipes and masks until (1) all citizens can correctly understand the microplastic pollution of discarded wipes and masks to the environment and consciously change their usual behavior, and correctly deal with the garbage; (2) these collected wipes and masks are properly disposed of without reentering the environment; (3) the government should actively encourage the development and production of degradable materials, so as to solve the environmental pollution caused by disposable wipes and masks; and (4) we should actively assess the potential environmental risks of wipes and masks that have entered the environment and the released microplastic fibers, and master the environmental behavior and fate of wipes and masks, so as to provide reference for future research.

Author contribution TH: writing-original draft; MS: writingreviewing and editing; WT: resources, conceptualization, writingreview and editing, supervision; all authors read and approved the final manuscript.

Funding The authors are grateful for the financial supports from the Innovative Province Construction of Hunan Province (2019RS1025), the Natural Science Foundation of Hunan Province (2021JJ30125), the Research and Development Plan of Key Areas in Hunan Province (2022SK2066), the Scientific Research Project of Hunan Provincial Education Department (20K032) and the National Natural Science Foundation of China (51809088).

Data availability All data generated or analyzed during this study are included in this published article (and its supplementary information files).

\section{Declarations}

Ethics approval and consent to participate Not applicable 
Consent for publication Not applicable

Competing interests The authors declare no competing interests.

\section{References}

Aragaw TA (2020) Surgical face masks as a potential source for microplastic pollution in the COVID-19 scenario. Mar Pollut Bull 159:111517

Belzagui F, Buscio V, Gutiérrez-Bouzán C, Vilaseca M (2021) Cigarette butts as a microfiber source with a microplastic level of concern. Sci Total Environ 762:144165

Bouwmeester H, Hollman PC, Peters RJ (2015) Potential health impact of environmentally released micro- and nanoplastics in the human food production chain: experiences from nanotoxicology. Environ Sci Technol 49:8932-8947

Briain OO, Marques Mendes AR, McCarron S, Healy MG, Morrison L (2020) The role of wet wipes and sanitary towels as a source of white microplastic fibres in the marine environment. Water Res 182:116021

Browne MA, Crump P, Niven SJ, Teuten E, Tonkin A, Galloway T et al (2011) Accumulation of microplastic on shorelines worldwide: sources and sinks. Environ Sci Technol 45:9175-9179

Cox KD, Covernton GA, Davies HL, Dower JF, Juanes F, Dudas SE (2019) Human consumption of microplastics. Environ Sci Technol 53:7068-7074

De Falco F, Gullo MP, Gentile G, Di Pace E, Cocca M, Gelabert L et al (2018) Evaluation of microplastic release caused by textile washing processes of synthetic fabrics. Environ Pollut 236:916-925

Enfrin M, Lee J, Gibert Y, Basheer F, Kong L, Dumee LF (2020) Release of hazardous nanoplastic contaminants due to microplastics fragmentation under shear stress forces. J Hazard Mater 384:121393

Everaert G, Van Cauwenberghe L, De Rijcke M, Koelmans AA, Mees J, Vandegehuchte M et al (2018) Risk assessment of microplastics in the ocean: modelling approach and first conclusions. Environ Pollut 242:1930-1938

Fadare OO, Okoffo ED (2020) Covid-19 face masks: a potential source of microplastic fibers in the environment. Sci Total Environ 737:140279

Gerd L, Elisabeth L (2013) Non-pollen particulates in honey and sugar. Food Addit Contam 30:2136-2140

Gerd L, Elisabeth L (2014) Synthetic particles as contaminants in German beers. Food Addit Contam 31:1574-1578

Han J, He S (2021) Need for assessing the inhalation of micro(nano) plastic debris shed from masks, respirators, and home-made face coverings during the COVID-19 pandemic. Environ Pollut 268:115728

Hodson ME, Duffus-Hodson CA, Clark A, Prendergast-Miller MT, Thorpe KL (2017) Plastic bag derived-microplastics as a vector for metal exposure in terrestrial invertebrates. Environ Sci Technol 51:4714-4721

Horton AA, Walton A, Spurgeon DJ, Lahive E, Svendsen C (2017) Microplastics in freshwater and terrestrial environments: evaluating the current understanding to identify the knowledge gaps and future research priorities. Sci Total Environ 586:127-141

Hu D, Shen M, Zhang Y, Li H, Zeng G (2019) Microplastics and nanoplastics: would they affect global biodiversity change? Environ Sci Pollut Res 26:19997-20002

Huang YQ, Wong CKC, Zheng JS, Bouwman H, Barra R, Wahlström B et al (2012) Bisphenol A (BPA) in China: a review of sources, environmental levels, and potential human health impacts. Environ Int 42:91-99
Iniguez ME, Conesa JA, Fullana A (2017) Microplastics in Spanish table salt. Sci Rep 7:8620

Khan A, Orr B, Joksimovic D (2019) Defining "flushability" for sewer use. Technical report by ryerson urban water center for municipal enforcement sewer use group of Canada. https://www.ryerson.ca/ content/dam/water/Research/FinalReport-FlushablesApril1.pdf

Kirstein IV, Kirmizi S, Wichels A, Garin-Fernandez A, Erler R, Löder $M$ et al (2016) Dangerous hitchhikers? Evidence for potentially pathogenic Vibrio spp. on microplastic particles. Mar Environ Res 120:1-8

Koelmans AA, Bakir A, Burton GA, Janssen CR (2016) Microplastic as a vector for chemicals in the aquatic environment. Critical review and model-supported re-interpretation of empirical studies. Environ Sci Technol 50:3315-3326

Koelmans AA, Besseling E, Wegner A, Foekema EM (2013) Plastic as a carrier of pops to aquatic organisms: a model analysis. Environ Sci Technol 47:7812-7820

Koelmans AA, Mohamed Nor NH, Hermsen E, Kooi M, Mintenig SM, De France J (2019) Microplastics in freshwaters and drinking water: critical review and assessment of data quality. Water Res 155:410-422

Lares M, Ncibi MC, Sillanpää M, Sillanpää M (2018) Occurrence, identification and removal of microplastic particles and fibers in conventional activated sludge process and advanced MBR technology. Water Res 133:236-246

Li J, Qu X, Su L, Zhang W, Yang D, Kolandhasamy P et al (2016) Microplastics in mussels along the coastal waters of China. Environ Pollut 214:177-184

Ma J, Chen F, Xu H, Jiang H, Liu J, Li P et al (2021) Face masks as a source of nanoplastics and microplastics in the environment: quantification, characterization, and potential for bioaccumulation. Environ Pollut 288:117748

Mintenig SM, Loder MGJ, Primpke S, Gerdts G (2019) Low numbers of microplastics detected in drinking water from ground water sources. Sci Total Environ 648:631-635

Murphy F, Ewins C, Carbonnier F, Quinn B (2016) Wastewater treatment works (wwtw) as a source of microplastics in the aquatic environment. Environ Sci Technol 50:5800-5808

Napper IE, Thompson RC (2016) Release of synthetic microplastic plastic fibres from domestic washing machines: effects of fabric type and washing conditions. Mar Pollut Bull 112:39-45

Novotny TE, Lum K, Smith E, Wang V, Barnes R (2009) Cigarettes butts and the case for an environmental policy on hazardous cigarette waste. Int J Environ Res Pub He 6:1691-1705

Oßmann BE, Sarau G, Holtmannspötter H, Pischetsrieder M, Christiansen SH, Dicke W (2018) Small-sized microplastics and pigmented particles in bottled mineral water. Water Res 141:307-316

Parker TT, Rayburn J (2017) A comparison of electronic and traditional cigarette butt leachate on the development of Xenopus laevis embryos. Toxicol Rep 4:77-82

Prata JC, Silva ALP, Walker TR, Duarte AC, Rocha-Santos T (2020) COVID-19 Pandemic repercussions on the use and management of plastics. Environ Sci Technol 54:7760-7765

Ragusa A, Svelato A, Santacroce C, Catalano P, Notarstefano V, Carnevali $\mathrm{O}$ et al (2021) Plasticenta: first evidence of microplastics in human placenta. Environ Int 146:106274

Rebelein A, Int-Veen I, Kammann U, Scharsack JP (2021) Microplastic fibers-underestimated threat to aquatic organisms? Sci Total Environ 777:146045

Saliu F, Veronelli M, Raguso C, Barana D, Galli P, Lasagni M (2021) The release process of microfibers: from surgical face masks into the marine environment. Environ Adv 4:100042

Schmidt N, Thibault D, Galgani F, Paluselli A, Sempere R (2018) Occurrence of microplastics in surface waters of the Gulf of Lion (NW Mediterranean Sea). Prog Oceanogr 163:214-220 
Schwabl P, Koeppel S, Koenigshofer P, Bucsics T, Trauner M, Reiberger T et al (2019) Detection of various microplastics in human stool a prospective case series. Ann Inter Med 171:453-457

Shen M, Song B, Zeng G, Zhang Y, Huang W, Wen X, Tang W (2020) Are biodegradable plastics a promising solution to solve the global plastic pollution? Environ Pollut 263:114469

Shen M, Song B, Zeng G, Zhang Y, Teng F, Zhou C (2021) Surfactant changes lead adsorption behaviors and mechanisms on microplastics. Chem Eng J 405:126989

Shen M, Zeng Z, Song B, Yi H, Hu T, Zhang Y et al (2021) Neglected microplastics pollution in global COVID-19: disposable surgical masks. Sci Total Environ 790:148130

Shen M, Zeng Z, Wen X, Ren X, Zeng G, Zhang Y et al (2021c) Presence of microplastics in drinking water from freshwater sources: the investigation in Changsha, China. Environ Sci Pollut Res 28:42313-42324

Shen M, Zhang Y, Zhu Y, Song B, Zeng G, Hu D et al (2019a) Recent advances in toxicological research of nanoplastics in the environment: a review. Environ Pollut 252:511-521

Shen M, Zhu Y, Zhang Y, Zeng G, Wen X, Yi H et al (2019b) Micro(nano)plastics: unignorable vectors for organisms. Mar Pollut Bull 139:328-331

Shen M, Zeng G, Zhang Y, Wen X, Song B, Tang W (2019) Can biotechnology strategies effectively manage environmental (micro) plastics? Sci Total Environ 697:134200

Shen M, Ye S, Zeng G, Zhang Y, Xing L, Tang W, Wen X, Liu S (2019) Can microplastics pose a threat to ocean carbon sequestration? Mar Pollut Bull 150:110712

Shruti VC, Pérez-Guevara F, Elizalde-Martínez I, Kutralam-Muniasamy G (2020) Reusable masks for COVID-19: a missing piece of the microplastic problem during the global health crisis. Mar Pollut Bull 161:111777

Sullivan GL, Delgado-Gallardo J, Watson TM, Sarp S (2021) An investigation into the leaching of micro and nano particles and chemical pollutants from disposable face masks-linked to the COVID-19 pandemic. Water Res 196:117033

Sun X, Liang J, Zhu M, Zhao Y, Zhang B (2018) Microplastics in seawater and zooplankton from the Yellow Sea. Environ Pollut 242:585-595

Sussarellu R, Suquet M, Thomas Y, Lambert C, Fabioux C, Pernet ME et al (2016) Oyster reproduction is affected by exposure to polystyrene microplastics. P Natl Acad SCI USA 113:2430

Thompson RC, Olsen Y, Mitchell RP, Davis A, Rowland SJ, John AWG et al (2004) Lost at sea: where is all the plastic? Science 304:838-838

Tien C, Wang Z, Chen C (2020) Microplastics in water, sediment and fish from the Fengshan River system: relationship to aquatic factors and accumulation of polycyclic aromatic hydrocarbons by fish. Environ Pollut 265:114962
Vandermeersch G, Cauwenberghe LV, Janssen CR, Marques A, Granby $\mathrm{K}$, Fait $\mathrm{G}$ et al (2015) A critical view on microplastic quantification in aquatic organisms. Environ Res 143:46-55

Velzeboer I, Kwadijk CJAF, Koelmans AA (2014) Strong sorption of PCBs to nanoplastics, microplastics, carbon nanotubes, and fullerenes. Environ Sci Technol 48:4869-4876

Vethaak AD, Leslie HA (2016) Plastic debris is a human health issue. Environ Sci Technol 50:6825-6826

Wang Z, An C, Chen X, Lee K, Zhang B, Feng Q (2021) Disposable masks release microplastics to the aqueous environment with exacerbation by natural weathering. J Hazard Mater 417:126036

Waring RH, Harris RM, Mitchell SC (2018) Plastic contamination of the food chain: a threat to human health? Maturitas 115:64-68

Woods MN, Stack ME, Fields DM, Shaw SD, Matrai PA (2018) Microplastic fiber uptake, ingestion, and egestion rates in the blue mussel (Mytilus edulis). Mar Pollut Bull 137:638-645

World Health Organization (2020) Shortage of personal protective equipment endangering health workers worldwide

Wright SL, Kelly FJ (2017) Plastic and human health: a micro issue? Environ Sci Technol 51:6634-6647

Wu P, Li J, Lu X, Tang Y, Cai Z (2021) Release of tens of thousands of microfibers from discarded face masks under simulated environmental conditions. Sci Total Environ 2021:150458

Xu C, Zhang B, Gu C, Shen C, Yin S, Aamir M et al (2020) Are we underestimating the sources of microplastic pollution in terrestrial environment? J Hazard Mater 400:123228

Xu E, Ren Z (2021) Preventing masks from becoming the next plastic problem. Front Environ Sci Eng 15:125

Yang D, Shi H, Li L, Li J, Jabeen K, Kolandhasamy P (2015) Microplastic pollution in table salts from China. Environ Sci Techno 149:13622-13627

Yang Y, Liu W, Zhang Z, Grossart H-P, Gadd GM (2020) Microplastics provide new microbial niches in aquatic environments. Appl Microbiol Biot 104:6501-6511

Yoo JW, Doshi N, Mitragotri S (2011) Adaptive micro and nanoparticles: temporal control over carrier properties to facilitate drug delivery. Adv Drug Deliver Rev 62:1247-1256

Zhang Y, Liang J, Zeng G, Tang W, Lu Y, Luo Y, Xing W et al (2020) How climate change and eutrophication interact with microplastic pollution and sediment resuspension in shallow lakes: a review. Sci Total Environ 705:135979

Publisher's note Springer Nature remains neutral with regard to jurisdictional claims in published maps and institutional affiliations. 